\title{
Galantamine protects against lipopolysaccharide- induced acute lung injury in rats
}

\author{
G. Li, C.L. Zhou, Q.S. Zhou and H.D. Zou \\ Department of Critical Care Medicine, Renmin Hospital, Wuhan University, Wuhan, Hubei Province, China
}

\begin{abstract}
Lipopolysaccharide (LPS)-induced endotoxemia triggers the secretion of proinflammatory cytokines and can cause acute lung injury (ALI). The high mobility group box 1 (HMGB1) protein plays an important role as a late mediator of sepsis and ALI. Galantamine (GAL) is a central acetylcholinesterase inhibitor that inhibits the expression of HMGB1. This study evaluated the effects of GAL by measuring levels of inflammatory mediators and observing histopathological features associated with LPSinduced ALI. Sixty 8-10 week old male Sprague-Dawley rats (200-240 g) were randomized into three groups as follows: control group, LPS group $(7.5 \mathrm{mg} / \mathrm{kg}$ LPS), and LPS + GAL group $(5 \mathrm{mg} / \mathrm{kg}$ GAL before LPS administration). Histopathological examination of lung specimens obtained $12 \mathrm{~h}$ after LPS administration was performed to analyze changes in wet-to-dry (W/D) weight ratio, myeloperoxidase (MPO) activity, and HMGB1 expression level. Additionally, plasma concentrations of tumor necrosis factor- $\alpha$, interleukin-6, and HMGB1 were measured using an enzyme-linked immunosorbent assay at 0 (baseline), 3,6 , 9 , and $12 \mathrm{~h}$ after LPS administration. Mortality in the three groups was recorded at $72 \mathrm{~h}$. LPS-induced ALI was characterized by distortion of pulmonary architecture and elevation of MPO activity, W/D weight ratio, and levels of pro-inflammatory cytokines, including tumor necrosis factor- $\alpha$, interleukin-6, and HMGB1. Pretreatment with GAL significantly reduced the LPS-induced lung pathological changes, W/D weight ratio, levels of pro-inflammatory cytokines and MPO activity (ANOVA). Moreover, GAL treatment significantly decreased the mortality rate (ANOVA). In conclusion, we demonstrated that GAL exerted a protective effect on LPS-induced ALI in rats.
\end{abstract}

Key words: Galantamine; Acute lung injury; Lipopolysaccharide; HMGB1

\section{Introduction}

Acute lung injury (ALI) is a leading cause of death in patients with sepsis, and has shown an annual increase in incidence over the past few years $(1,2)$. Despite remarkable advances in sepsis treatment, the occurrence of ALI and subsequent acute respiratory failure in critically ill patients remains unacceptably high (3).

The exact nature of the cell signaling pathways involved in the pathophysiology of sepsis-induced ALI remains elusive. However, cumulative evidence has suggested that the process is mediated by increased pulmonary expression of pro-inflammatory cytokines, such as tumor necrosis factor (TNF)- $\alpha$, interleukin (IL)-1, IL-6, and high mobility group box 1 (HMGB1) (4). These pro-inflammatory cytokines are believed to trigger, amplify, and perpetuate the inflammatory response, thereby affecting gas exchange and causing refractory hypoxemia.

HMGB1 is an intranuclear protein that was initially recognized to be crucial for the regulation of gene transcription and stabilization of the nucleosome. Extracellular HMGB1 released from necrotic tissue and activated monocytes and macrophages functions as a late mediator in $A L I$ secondary to sepsis. HMGB1 is reported to be involved in neutrophil accumulation, interstitial edema, disruption of epithelial integrity, leakage of proteins into the alveolar space, and increased production of pro-inflammatory cytokines associated with the pathogenesis of $\operatorname{ALI}(5,6)$. In addition, anti-HMGB1 antibodies prevented death in experimental mice with sepsis and the resultant $\operatorname{ALI}(7,8)$, indicating that therapeutic agents that attenuate HMGB1 release may have potential for the prevention and treatment of ALI.

Galantamine (GAL) is a competitive and reversible cholinesterase inhibitor that is used in the management of Alzheimer's disease and other conditions involving memory impairment $(9,10)$. Recent studies showed that GAL attenuated the severity of local inflammation in animals, suppressed the degree of systemic inflammatory response elicited by lipopolysaccharide (LPS), and inhibited TNF- $\alpha$ expression in rats with LPS-induced peritonitis $(11,12)$. The anti-inflammatory action of GAL might be mediated by the cholinergic nervous system, which exerts its effects via the vagus nerve and functions as a natural

Correspondence: G. Li: <9620594@qq.com>

Received June 7, 2015 | Accepted September 28, 2015 
anti-inflammatory system to prevent the excessive release of inflammatory cytokines in the event of infection, sepsis, or autoimmune diseases such as rheumatoid arthritis $(13,14)$. Recent studies have shown that the nicotinic acetylcholine receptor alpha7 subunit plays an important role in the cholinergic anti-inflammatory effect (15). GAL, which is extracted from the bulb of the snowdrop flower, is an agonist to nicotinic acetylcholine receptors, and therefore is expected to enhance the cholinergic anti-inflammatory pathway. However, it remains to be determined whether GAL can exert anti-inflammatory effects to reduce the severity of ALI secondary to sepsis.

The present study investigated whether GAL inhibits the production of inflammatory cytokines and reduces the severity of LPS-induced ALI.

\section{Material and Methods}

\section{Ethics statement}

All of the experimental procedures performed in this study were in accordance with the Guide for the Care and Use of Laboratory Animals, proposed by the National Institutes of Health. The study protocol was approved by the animal experimental Ethics Committee of Wuhan University, China.

\section{Experiment animals}

A total of 90 8-10 week old male Sprague-Dawley rats $(200-240 \mathrm{~g})$ were used in this study. All experimental animals were obtained from the Experiment Animal Center, Wuhan University (permit number: Hubei 00001306). The rats were housed in cages maintained at room temperature with a 12-h light/dark cycle. They were fed with standard pellet diet and tap water ad libitum.

\section{Reagents}

Escherichia coli 0111:B4 endotoxin was purchased from Sigma-Aldrich (USA). GAL was purchased from EMD Biosciences Inc. (USA). The rabbit anti-HMGB1 polyclonal antibody was obtained from Boster Biotechnology Co. (China), and antibodies for Western blotting were purchased from Pierce (Pierce Biotechnology, USA). The kit to determine HMGB1 expression using the streptavidinbiotic complex method was obtained from Boster Biotechnology Co. The myeloperoxidase (MPO) activity kit was obtained from Jiancheng Bioengineering Institute (China) and the cytokine immunoassay kits were purchased from R\&D Systems (USA).

\section{Experimental protocols}

Rats were randomized into three groups: LPS group $(n=30)$, in which LPS $(7.5 \mathrm{mg} / \mathrm{kg}$, dissolved in $0.5 \mathrm{~mL}$ sterile saline) was administered by an intravenous (iv) injection via the tail vein; LPS + GAL group $(n=30)$, in which GAL (5 mg/kg, intraperitoneal, ip) was administered $30 \mathrm{~min}$ before injection of LPS $(7.5 \mathrm{mg} / \mathrm{kg}$, dissolved in $0.5 \mathrm{~mL}$ sterile saline, iv); and a control group $(n=30)$, in which the rats were administered saline at a volume equivalent to that in the other groups. Ten rats in each group were separately investigated as a subgroup for survival analysis. Rats were euthanized with an overdose of sodium pentobarbital (100 mg/kg, ip). Then, lung tissue specimens and blood samples were obtained for further analysis.

\section{Survival study}

To determine the mortality rate, the survival rate in all three groups was assessed at $72 \mathrm{~h}$ after the administration of LPS.

\section{Histologic analysis}

Twelve hours after LPS administration, the rats were euthanized ( $n=5,3$, and 5 in the control, LPS, and LPS + GAL groups, respectively). The obtained lung tissue specimens were fixed with $10 \%$ formalin, embedded in paraffin, cut into $5 \mu \mathrm{m}$-thick sections and mounted onto slides. The sections were then stained with hematoxylin and eosin (H\&E) as per the standard staining method (16). Histologic changes were graded by a pathologist blinded to the clinical status of the rats. The lung tissue samples were then scored for the degree of intra-alveolar edema, intra-alveolar hemorrhage, and neutrophil infiltration using grades 0 to 4 (0, absent; 1 , mild; 2, moderate; 3 , severe; 4 , overwhelming) with a maximum score of 12 , as described previously (17).

\section{Wet-to-dry weight ratio}

After the animals were euthanized at $12 \mathrm{~h}$, the chest cavity was opened and the right lung was ligated and excised. The lung specimen was then rinsed briefly in phosphate buffered saline (PBS), blotted, and weighed to determine the 'wet' weight. Subsequently, the lungs were dried in an oven at $80^{\circ} \mathrm{C}$ for $48 \mathrm{~h}$ to obtain the 'dry' weight. The ratio of wet-to-dry (W/D) weight was then calculated.

\section{MPO assay}

The level of MPO activity in the lung parenchyma, which is a marker of the extent of neutrophil infiltration, was measured $12 \mathrm{~h}$ after LPS administration, by using a modified version of a previously described method (18). In brief, frozen lung specimens were weighed and homogenized in hexadecyltrimethylammonium bromide (HTAB) buffer (0.5\% HTAB in $50 \mathrm{mM}$ phosphate buffer, pH 6.0). The supernatant of the homogenate was then collected after sonication and centrifugation at $40,000 \mathrm{~g}$ for $15 \mathrm{~min}$. MPO activity was determined by measuring the $\mathrm{H}_{2} \mathrm{O}_{2}$-dependent oxidation of o-dianisidinehydrochloride in a 96-well plate reader, at $460 \mathrm{~nm}$. MPO activity was expressed per gram of lung weight.

\section{Immunohistochemical analysis for HMGB1}

The lung sections were immunostained for HMGB1 using the streptavidin-biotin complex. All steps, including 
deparaffinization and counterstaining with hematoxylin, were performed in accordance with the manufacturer's instructions. Non-specific binding of the antibodies was blocked by incubating them with $100 \mu \mathrm{L}$ of $5 \%$ normal goat serum for $20 \mathrm{~min}$. Then, the lung sections were incubated overnight at $4^{\circ} \mathrm{C}$ in the presence of mouse anti-rat HMGB1 polyclonal antibody (1:1000) as the primary antibody, washed with phosphate buffered saline, and incubated in the presence of anti-mouse $\operatorname{lgG}$ antibody $(1: 1000)$ as the secondary antibody. The number of brown granules in each highpowered field (magnification: $\times 400$ ) was quantified as the number of positively stained cells or nuclei. The measurements were expressed as the percentage of positively stained cells or the ratio of nuclei to the total cells observed in 8-10 digital images per animal.

\section{Western blot \\ Protein extracts obtained from the lung tissues were obtained $12 \mathrm{~h}$ after LPS administration. Western blot analysis of the extracts was performed as previously described (19). In brief, the membranes were incubated for $1 \mathrm{~h}$ at room temperature in the presence of the primary antibody (dilution, 1:1000), washed three times with TTBS buffer, and incubated at room temperature for $1 \mathrm{~h}$ in the presence of the secondary antibody (dilution, 1:5000). After the blots were washed three times with TTBS buffer, they were developed and exposed by enhanced chemiluminescence.}

Plasma levels of cytokines (TNF- $\alpha$, IL-6 and HMGB1)

Blood samples were collected via cardiac puncture at $3,6,9$, and $12 \mathrm{~h}$ after the administration of LPS. All rats were euthanized with phenobarbital sodium before blood collection. The collected blood samples were centrifuged at $377.3 \mathrm{~g}$ for $10 \mathrm{~min}$ at $4^{\circ} \mathrm{C}$, and the plasma supernatant retrieved was stored at $-20^{\circ} \mathrm{C}$ until further analysis. The plasma levels of TNF- $\alpha$ and IL- 6 were detected using solid-phase sandwich enzyme-linked immunosorbent assay (ELISA) kits specific for the detection of these factors, and the absorbance was measured at $450 \mathrm{~nm}$ by a plate reader (BioTek ELx800, USA).

\section{Statistical analysis}

Data are reported as the means \pm SE or SD. Intergroup comparisons were made using one-way analysis of variance (ANOVA), followed by Student-Newman-Keuls q-test (SNK-q). The differences were considered to be significant if $\mathrm{P}<0.05$.

\section{Results}

\section{Effect of GAL on LPS-induced mortality}

Ten rats in each group were investigated for survival analysis at $72 \mathrm{~h}$ after LPS injection. As expected, all the rats in the control group survived. However, in the LPS group, $50 \%$ of the rats died within $24 \mathrm{~h}$ while an additional $30 \%$ died within $72 \mathrm{~h}$; thus, the total mortality rate in this group was $80 \%$ in contrast to the LPS + GAL group where the mortality rate was $10 \%$.

\section{GAL pre-treatment attenuated LPS-induced pathological changes in lung tissue}

The control group showed no significant histological alterations. The rats exposed to LPS showed increased alveolar wall thickness, edema, bleeding, and infiltration of inflammatory cells at $12 \mathrm{~h}$ after LPS administration, indicating the occurrence of ALI. Rats pre-treated with GAL showed significantly less inflammation and distortion of pulmonary architecture after LPS administration as compared to those not treated with GAL. (Figure 1A-C; H\&E staining, $\times 200$ magnification). The total scores of the pulmonary histological changes in the groups indicated that the degree of pulmonary injury in the LPS + GAL group was significantly less than that in the LPS group $(P<0.05$, Figure 1D).

\section{Effect of GAL pre-treatment on W/D ratio}

The LPS group had a significantly higher W/D ratio than the control group, indicating the presence of pulmonary edema $(P<0.05)$. However, the W/D ratio in the $L P S+G A L$ group was significantly lower than that in the LPS group, indicating that GAL attenuated the degree of pulmonary edema induced by LPS $(P<0.05$, Figure 2$)$.

\section{Effect of GAL on LPS-induced MPO activity}

The level of MPO activity in the LPS group was significantly higher than that in the control group $(10.2 \pm 1.12$ vs $1.8 \pm 0.35 \mathrm{U} / \mathrm{g}$ tissue, $\mathrm{P}<0.01)$. However, the MPO activity level in the LPS + GAL group was significantly lower than that in the LPS group $(3.8 \pm 0.62 \mathrm{U} / \mathrm{g}$ tissue, $\mathrm{P}<0.05$ vs the LPS group), indicating that GAL inhibited MPO activity (Figure 3 ).

\section{GAL suppressed LPS-induced HMGB1 expression in lungs}

At $12 \mathrm{~h}$, the expression levels of HMGB1 protein in the LPS group were considerably higher than those in the control group, while the expression levels in the LPS + GAL group were markedly lower than those in the LPS group (Figure 4). This indicated that GAL down-regulated the LPSinduced increase in HMGB1 expression, similar to that indicated by the Western blot analysis (Figure 5).

\section{GAL down-regulated the release of pro-inflammatory cytokines}

In the LPS group, the levels of TNF- $\alpha$ and IL- 6 increased sharply after LPS administration and reached peak levels at $3 \mathrm{~h}$. Thereafter, the levels decreased gradually to baseline levels at $12 \mathrm{~h}$. However, the levels of the late stage proinflammatory cytokine HMGB1 increased gradually and reached a peak at $12 \mathrm{~h}$. In contrast, the rats pretreated with GAL had significantly lower levels of TNF- $\alpha$ (LPS + GAL group vs LPS group: $\mathrm{P}<0.05$ at 3 and $6 \mathrm{~h}$ ), IL-6 

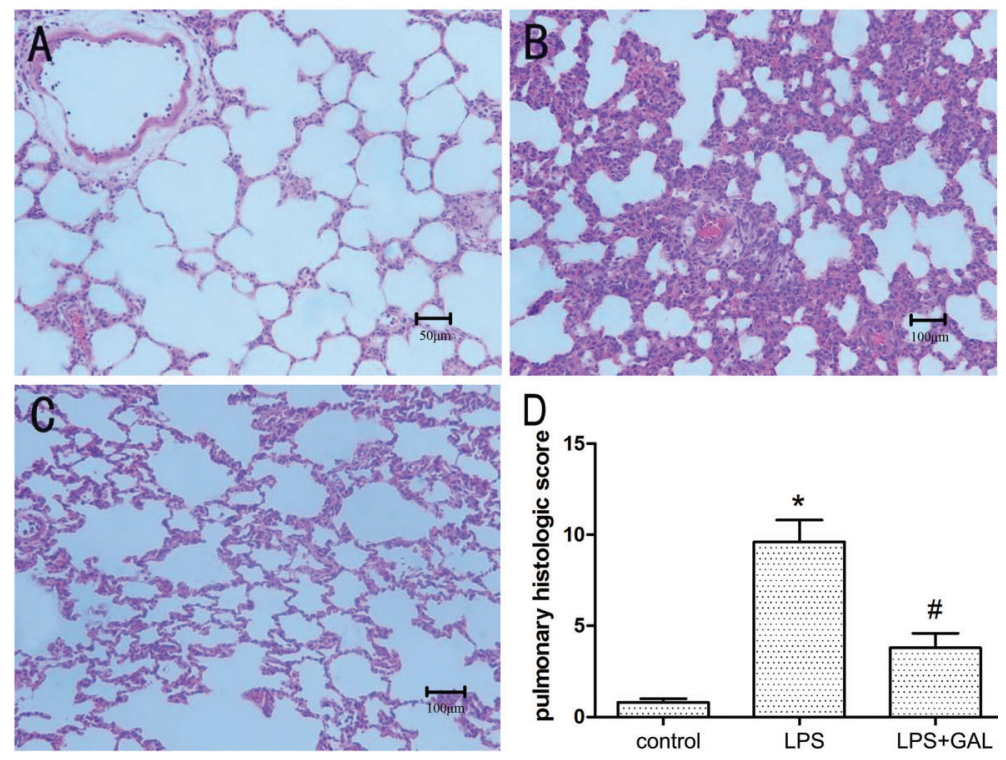

Figure 1. Histopathological changes in lung tissue samples of the three groups. Hematoxylin and eosin stain ( $\times 200$ magnification). A, Control group $(\mathrm{n}=5)$ : normal lung structure (bar $50 \mu \mathrm{m})$. $B$, LPS group $(n=3)$ : increased alveolar wall thickness, edema, bleeding, and infiltration of inflammatory cells (bar $100 \mu \mathrm{m}) . C$, LPS + GAL group $(\mathrm{n}=5)$ : mild structure destruction and inflammatory infiltration. (bar $100 \mu \mathrm{m}$ ). $D$, comparison of the pulmonary histological scores of the 3 groups. GAL: galantamine; LPS: lipopolysaccharide. ${ }^{*} \mathrm{P}<0.05$, LPS group compared to control group; ${ }^{*} P<0.05$, LPS + GAL group compared to LPS group (ANOVA).

(LPS + GAL group vs LPS group: $\mathrm{P}<0.05$ at 3, 6 and $9 \mathrm{~h}$ ), and HMGB1 (LPS + GAL group vs LPS group: $\mathrm{P}<0.05$ at 6,9 and $12 \mathrm{~h}$ ) at the indicated time points (Figure 6).

\section{Discussion}

In the present study, a rat model of ALI was successfully established by the intravenous administration of LPS. We found that LPS exposure caused a dramatic increase in the MPO activity level and W/D ratio, reflecting the occurrence of neutrophil infiltration and pulmonary edema. Furthermore, histopathological analysis revealed the loss of epithelial integrity. Taken together, these manifestations

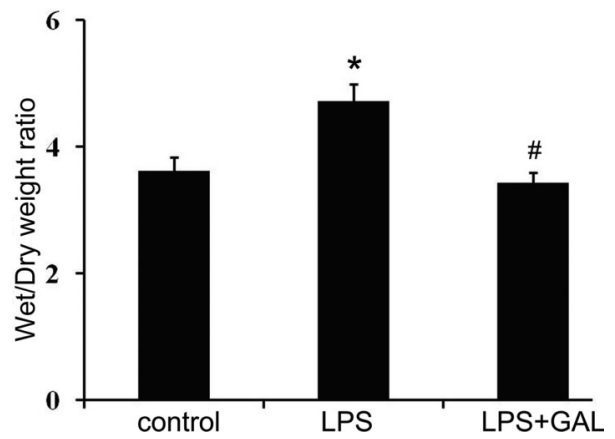

Figure 2. Comparison of the wet/dry weight ratio. The extent of pulmonary edema was assessed using the wet/dry ratio at $12 \mathrm{~h}$ after lipopolysaccharide (LPS) infusion. Control group: $n=5$; LPS group: $n=3$; LPS + galantamine (GAL) group: $n=5$. Data are reported as the means $\pm S D$. ${ }^{*} P<0.05$, LPS group compared to control group; ${ }^{\#} \mathrm{P}<0.05$, LPS $+\mathrm{GAL}$ group compared to LPS group (ANOVA). confirmed the development of LPS-induced ALI. Interestingly, pretreatment with GAL not only improved the survival of LPS-exposed rats, but also reduced the extent of histopathological changes, neutrophil infiltration, and secretion of proinflammatory cytokines in rat lung tissue.

Gram-negative sepsis is the most common risk factor of acute respiratory distress syndrome. LPS is the principal component of the outer membrane of gram-negative bacteria and is a potent stimulator of rapid pro-inflammatory cytokine production. The elevated expression of TNF- $\alpha$, IL-1 $\beta$, and IL- 6 is an important step in the pathogenesis of $\mathrm{ALI}$ and acute respiratory distress syndrome (20). Moreover, in the case of humans with ALI or sepsis, a persistent

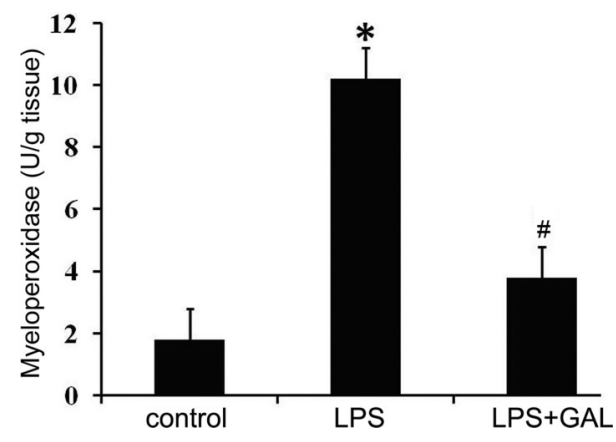

Figure 3. Effect of galantamine (GAL) on myeloperoxidase (MPO) activity in rat lungs. Neutrophil infiltration was assessed in terms of MPO activity level at $12 \mathrm{~h}$ after lipopolysaccharide (LPS) administration. Control group: $n=5$; LPS group: $n=3$; LPS + GAL group: $n=5$. Data are reported as the means $\pm S D$. ${ }^{*} \mathrm{P}<0.05$, LPS group compared to control group; ${ }^{*} \mathrm{P}<0.05$, LPS + GAL group compared to LPS group (ANOVA). 

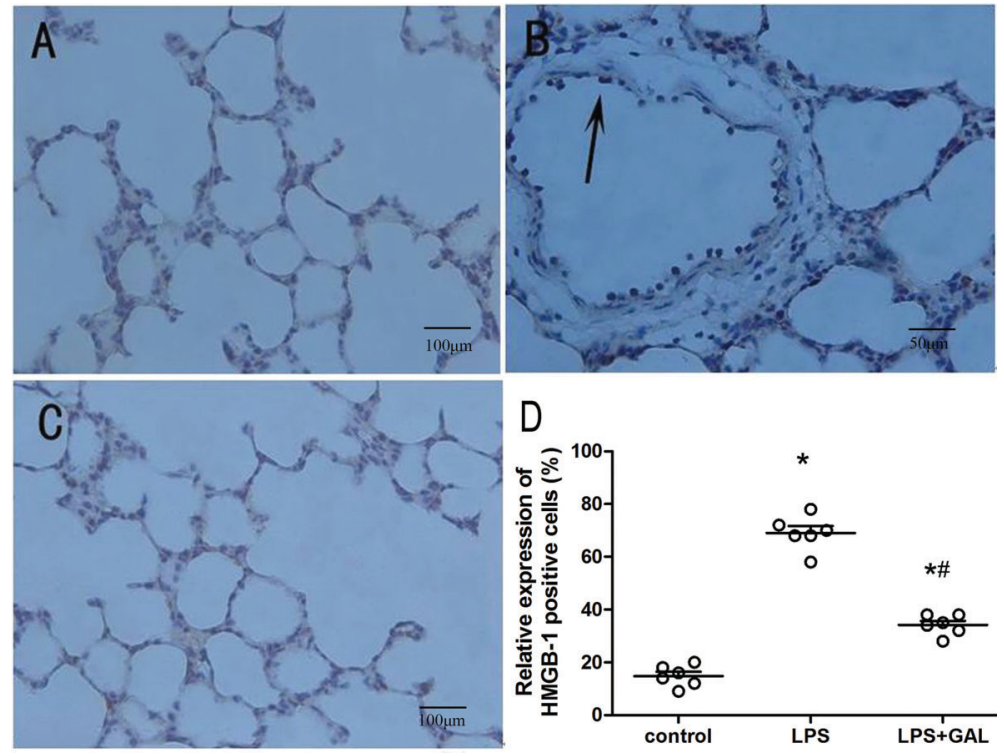

Figure 4. Immunohistochemical expression of high-mobility group box 1 (HMGB1) protein in rat lungs. $A$, Control group: $\mathrm{n}=5$ (bar $100 \mu \mathrm{m}$ ). $B$, lipopolysaccharide (LPS) group: $\mathrm{n}=3$ (bar $50 \mu \mathrm{m}$ ). C, LPS + galantamine (GAL) group: $\mathrm{n}=5$ (bar $100 \mu \mathrm{m}$ ). The arrow indicates cells that stained positive for HMGB1 expression. Representative photomicrographs of lung immunohistochemical analysis $(400 \times)$ show the increased redistribution of HMGB-1 expression from the nucleus to the cytoplasm and extracellular areas in bronchial epithelial cells, alveolar epithelial cells, and inflammatory cells. $D$, Scatter plot of HMGB-1-positive $(+)$ cells $(\%)$ in lung tissues. ${ }^{*} P<0.05$, LPS and LPS + GAL groups compared to control group; ${ }^{\#} \mathrm{P}<0.05$, LPS $+\mathrm{GAL}$ group compared to LPS group (ANOVA test). elevation of these cytokines was associated with a poor prognosis (21). However, previous clinical studies have shown that anti-inflammatory agents such as monoclonal anti-TNF antibodies, IL-1 receptor antagonists, and TNFreceptor fusion proteins fail to prolong patient survival $(22,23)$. This failure may be explained by the fact that the levels of TNF- $\alpha$ and IL-1 $\beta$ are elevated during the early stage of sepsis and recover at the late stage of disease. Consistently, our study showed that the levels of TNF- $\alpha$ and IL-6 reached a peak at $3 \mathrm{~h}$ after LPS administration and then returned to baseline levels thereafter. The persistence of lung injury suggests that other late stage downstream pro-inflammatory cytokines may be involved in the progression of ALI.

HMGB1 has been reported to play a crucial role in the inflammatory response and pathogenesis of LPS-induced lung injury (24). HMGB1 is a nuclear protein that functions as a DNA chaperone protein in normal cells and promotes interactions between proteins and DNA. In addition, HMGB1 is thought to be a late mediator of sepsis. In the current study, we observed a delayed elevation of HMGB1 in contrast to TNF- $\alpha$ and IL- 6 . This finding is consistent with those of a previous study, which showed that bile TNF- $\alpha$ concentrations peaked at $3 \mathrm{~h}$ after LPS challenge while HMGB1 concentrations showed a significant increase from 8 to $12 \mathrm{~h}(8)$. Furthermore, the intratracheal administration of HMGB1 induced neutrophil infiltration in lung tissues and increased the pulmonary expressions of pro-inflammatory cytokines such as TNF- $\alpha, \mathrm{IL}-1 \beta$, and macrophage inflammatory protein-2 (7). However, in the present study, we did not observe an increase in TNF- $\alpha$ and IL-6 levels subsequent to the increase in HMGB1 expression. This may be due to several reasons. First, changes in the cytokine levels were monitored only for
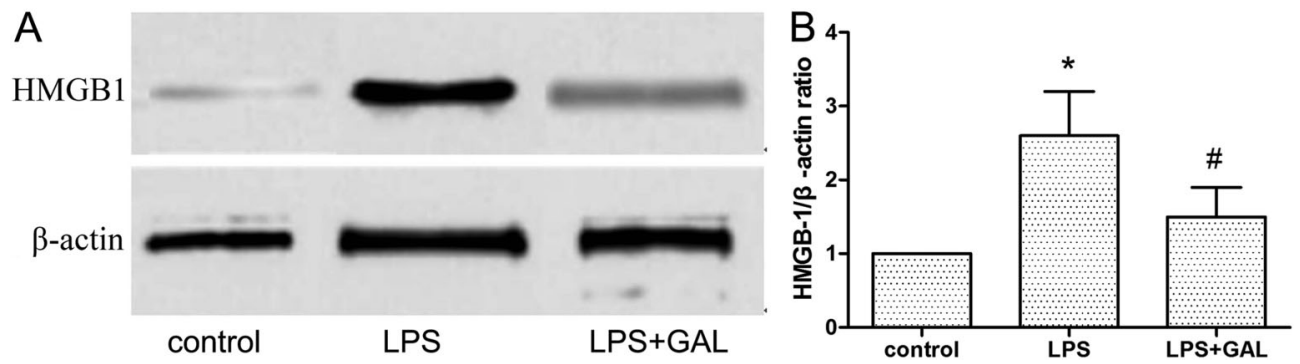

Figure 5. Western blot analysis of high-mobility group box 1 (HMGB1) levels in rat lung tissue at $12 \mathrm{~h}$. $A$, The concentrations of HMGB1 and $\beta$-actin in lung tissue were determined by Western blot analysis at $12 \mathrm{~h}$. Results of a representative experiment are shown. $B$, Galantamine (GAL) down-regulated the lipopolysaccharide (LPS)-induced elevation of HMGB1 expression. The results show the HMGB1/ $\beta$-actin ratio obtained from the Western blots. Control group: $n=5$; LPS group: $n=3$; LPS + GAL group: $n=5$. Data are reported as the means \pm SE. ${ }^{*} P<0.05$, LPS group compared to control group; ${ }^{\#} \mathrm{P}<0.05$, LPS + GAL group compared to LPS group (ANOVA). 

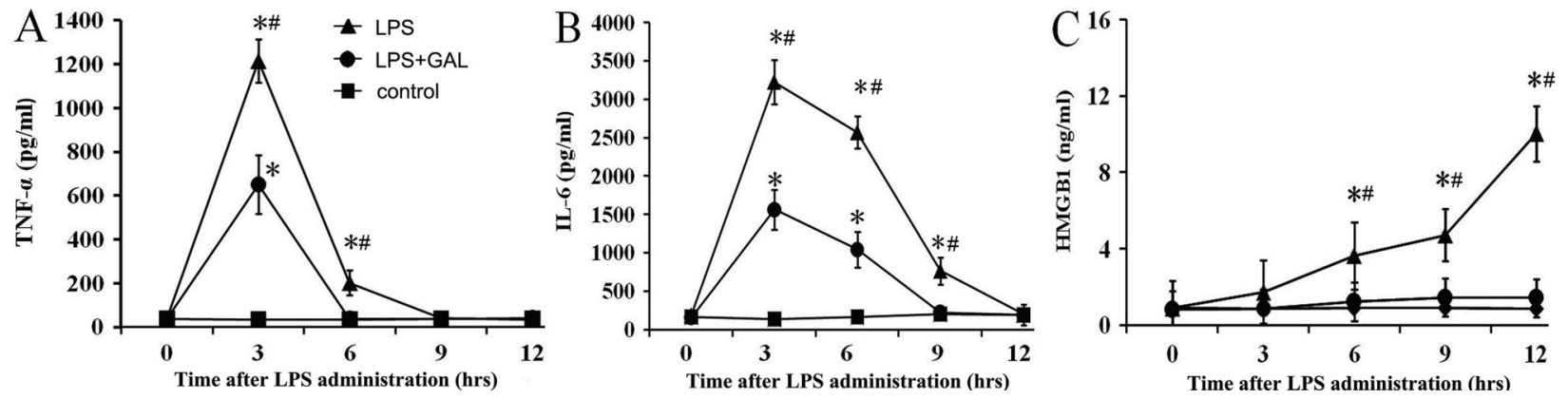

Figure 6. Changes in the levels of pro-inflammatory cytokines: $A$, tumor necrosis factor (TNF)- $\alpha, B$, interleukin (IL)-6, and $C$, highmobility group box 1 (HMGB1). Control group: $n=5$ for each time point; LPS group: $n=5$ ( 3 and $6 h), n=4$ ( $9 h$ ) and $n=3(12 h) ; L P S+G A L$ group: $n=5$ for each time point. GAL: galantamine; LPS: lipopolysaccharide. Data are reported as the means $\pm S E$. ${ }^{*} P<0.05$, LPS and LPS + GAL groups compared to control group; ${ }^{\#} \mathrm{P}<0.05$, LPS group compared to LPS + GAL group (ANOVA).

$12 \mathrm{~h}$ in this study. Therefore, it is not possible to comment whether fluctuations in the TNF- $\alpha$ and IL- 6 levels occurred after this time point. Second, it is unclear whether the amount of circulating HMGB1 generated in this study was sufficient to enhance the expression of other inflammatory cytokines.

Our findings indicated that GAL pretreatment inhibited HMGB1 expression in the plasma and lung tissues. GAL may thus mitigate the inflammatory response and lung injury symptoms through its inhibitory effect on HMGB1 levels in rats. Recent studies have shown that extracellular HMGB1 participated in signaling via receptor for advanced glycated end products (RAGE) and/or members of the toll-like receptor (TLR) family, namely, TLR2 and TLR4. RAGE was identified as the major functional receptor involved in the pro-inflammatory effect of HMGB1 in rodent macrophages. Furthermore, the activation of RAGE and TLR triggers inflammatory responses mediated by nuclear factor (NF)- $\kappa B$. Because TLR4 recognizes LPS from gram-negative bacilli, the interactions between HMGB1 and TLR4 may explain how HMGB1 triggers inflammatory responses similar to those elicited by LPS. In addition, studies have shown that anti-HMGB1 antibodies can prevent death in animal models of sepsis, hepatic ischemia-reperfusion injury, and rheumatoid arthritis $(25,26)$. This beneficial effect can be explained because the administration of anti-HMGB1 antibodies before or after endotoxin treatment causes a significant decrease in the extent of endotoxin-induced neutrophil infiltration and lung edema (20). Thus, our findings confirmed the anti-inflammatory effect of GAL. However, further studies are necessary to identify the downstream mechanisms.

Because MPO is mainly expressed in the primary granules of neutrophils, elevated levels of MPO levels in tissues imply the presence of neutrophil infiltration within lung parenchyma or alveolar spaces (27). In this study, we observed that GAL pretreatment led to a significant suppression of MPO activity in lung tissues, suggesting that GAL inhibited the neutrophil infiltration into the lung parenchyma and alveolar spaces in the setting of LPS-induced ALI.

The most common adverse effects reported with the use of GAL are gastrointestinal disturbances, such as nausea, vomiting, diarrhea, anorexia, and abdominal pain. However, these effects have been reported for the oral administration of GAL and not when administered as a single ip injection, as used in this study. The current study demonstrated that GAL elicited the following changes in rats with $\mathrm{ALI}$ : 1) improved early-stage survival rate, 2) ameliorated histopathological changes that indicate lung injury, and 3) inhibited the release of pro-inflammatory cytokines. Taken together, these results suggest that GAL might be a potential candidate for the treatment of LPS-induced ALI.

This study had some limitations. First, the effect of GAL was assessed for a short duration only. Second, the protocol in this study involved pretreatment of the rats with GAL before the administration of LPS; this is not consistent with disease in clinical settings. Third, this study did not clarify whether the continuous administration of GAL enhanced its protective effect. Thus, long-term investigations designed in accordance with clinical settings are necessary to verify the potential of GAL for the management of sepsisinduced ALI.

In conclusion, the current study showed that GAL exerted a protective effect against LPS-induced ALI, which appeared to be mediated by inhibiting the release of proinflammatory cytokines, especially HMGB1.

\section{Acknowledgments}

We appreciate the language editing and proofreading by Medjaden Bioscience Limited. 


\section{References}

1. Rubenfeld GD, Caldwell E, Peabody E, Weaver J, Martin DP, Neff $M$, et al. Incidence and outcomes of acute lung injury. N Engl J Med 2005;353:1685-93.

2. Mikkelsen ME, Shah CV, Meyer NJ, Gaieski DF, Lyon S, Miltiades AN, et al. The epidemiology of acute respiratory distress syndrome in patients presenting to the emergency department with severe sepsis. Shock 2013; 40: 375-81.

3. Ware LB, Matthay MA. The acute respiratory distress syndrome. N Engl J Med 2000; 342: 1334-1349.

4. Goodman RB, Pugin J, Lee JS, Matthay MA. Cytokinemediated inflammation in acute lung injury. Cytokine Growth Factor Rev 2003; 14: 523-535, doi: 10.1016/S1359-6101 (03)00059-5.

5. Kim JY, Park JS, Strassheim D, Douglas I, Diaz del Valle F, Asehnoune K, et al. HMGB1 contributes to the development of acute lung injury after hemorrhage. Am J Physiol Lung Cell Mol Physiol 2005; 288: L958-L965, doi: 10.1152/ ajplung.00359.2004.

6. Yang R, Miki K, Oksala N, Nakao A, Lindgren L, Killeen ME, et al. Bile high-mobility group box 1 contributes to gut barrier dysfunction in experimental endotoxemia. Am J Physiol Regul Integr Comp Physiol 2009; 297: R362-R369, doi: 10.1152/ajpregu.00184.2009.

7. Abraham E, Arcaroli J, Carmody A, Wang H, Tracey KJ. HMG-1 as a mediator of acute lung inflammation. J Immunol 2000; 165: 2950-2954, doi: 10.4049/jimmunol.165.6.2950.

8. Yang H, Ochani M, Li J, Qiang X, Tanovic M, Harris HE, et al. Reversing established sepsis with antagonists of endogenous high-mobility group box 1 . Proc Natl Acad Sci U S A 2004; 101: 296-301, doi: 10.1073/pnas.2434651100.

9. Pepeu G, Giovannini MG. Cholinesterase inhibitors and beyond. Curr Alzheimer Res 2009; 6: 86-96, doi: 10.2174/ 156720509787602861.

10. Fisher A. Cholinergic treatments with emphasis on $m 1$ muscarinic agonists as potential disease-modifying agents for Alzheimer's disease. Neurotherapeutics 2008; 5: 433-442, doi: 10.1016/j.nurt.2008.05.002.

11. Pavlov VA, Parrish WR, Rosas-Ballina M, Ochani M, Puerta M, Ochani $\mathrm{K}$, et al. Brain acetylcholinesterase activity controls systemic cytokine levels through the cholinergic anti-inflammatory pathway. Brain Behav Immun 2009; 23: 41-45, doi: 10.1016/j.bbi.2008.06.011.

12. Liu ZH, Ma YF, Wu JS, Gan JX, Xu SW, Jiang GY. Effect of cholinesterase inhibitor galanthamine on circulating tumor necrosis factor alpha in rats with lipopolysaccharide-induced peritonitis. Chin Med J 2010; 123: 1727-1730.

13. Huston JM. The vagus nerve and the inflammatory reflex: wandering on a new treatment paradigm for systemic inflammation and sepsis. Surg Infect 2012; 13: 187-193, doi: 10.1089/sur.2012.126.

14. Bruchfeld A, Goldstein RS, Chavan S, Patel NB, RosasBallina M, Kohn N, et al. Whole blood cytokine attenuation by cholinergic agonists ex vivo and relationship to vagus nerve activity in rheumatoid arthritis. J Intern Med 2010; 268: 94-101.
15. Gowayed MA, Refaat R, Ahmed WM, El-Abhar HS. Effect of galantamine on adjuvant-induced arthritis in rats. Eur J Pharmacol 2015; 764: 547-553.

16. Imanaka H, Shimaoka M, Matsuura N, Nishimura M, Ohta N, Kiyono $\mathrm{H}$. Ventilator-induced lung injury is associated with neutrophil infiltration, macrophage activation, and TGF-beta 1 mRNA upregulation in rat lungs. Anesth Analg 2001; 92: 428-436, doi: 10.1213/00000539-200102000-00029.

17. Chen F, Liu Z, Wu W, Rozo C, Bowdridge S, Millman A, et al. An essential role for $\mathrm{TH} 2$-type responses in limiting acute tissue damage during experimental helminth infection. Nat Med 2012; 18: 260-266, doi: 10.1038/nm.2628.

18. Goldblum SE, Wu KM, Jay M. Lung myeloperoxidase as a measure of pulmonary leukostasis in rabbits. $J$ Appl Physiol 1985; 59: 1978-1985.

19. Hagiwara S, Iwasaka H, Togo K, Noguchi T. A neutrophil elastase inhibitor, sivelestat, reduces lung injury following endotoxin-induced shock in rats by inhibiting HMGB1. Inflammation 2008; 31: 227-234, doi: 10.1007/s10753-0089069-z.

20. Giebelen IA, van Westerloo DJ, LaRosa GJ, de Vos AF, van der Poll T. Local stimulation of alpha7 cholinergic receptors inhibits LPS-induced TNF-alpha release in the mouse lung. Shock 2007; 28: 700-703.

21. Ghosh S, Latimer RD, Gray BM, Harwood RJ, Oduro A. Endotoxin-induced organ injury. Crit Care Med 1993; 21: S19-S24, doi: 10.1097/00003246-199302001-00005.

22. Abraham E, Anzueto A, Gutierrez G, Tessler S, San PG, Wunderink $R$, et al. Double-blind randomised controlled trial of monoclonal antibody to human tumour necrosis factor in treatment of septic shock. NORASEPT II Study Group. Lancet 1998; 351: 929-933, doi: 10.1016/S0140-6736(05) 60602-2.

23. Fisher CJ Jr, Dhainaut JF, Opal SM, Pribble JP, Balk RA, Slotman GJ, et al. Recombinant human interleukin 1 receptor antagonist in the treatment of patients with sepsis syndrome. Results from a randomized, double-blind, placebo-controlled trial. Phase III rhIL-1ra Sepsis Syndrome Study Group. JAMA 1994; 271: 1836-1843, doi: 10.1001/ jama.1994.03510470040032.

24. Ueno H, Matsuda T, Hashimoto S, Amaya F, Kitamura $Y$, Tanaka M, et al. Contributions of high mobility group box protein in experimental and clinical acute lung injury. Am J Respir Crit Care Med 2004; 170: 1310-1316, doi: 10.1164/rccm.200402-188OC.

25. Andersson U, Tracey KJ. HMGB1 as a mediator of necrosisinduced inflammation and a therapeutic target in arthritis. Rheum Dis Clin North Am 2004; 30: 627-637, xi, doi: 10.1016/j.rdc.2004.04.007.

26. Tsung A, Sahai R, Tanaka H, Nakao A, Fink MP, Lotze MT, et al. The nuclear factor HMGB1 mediates hepatic injury after murine liver ischemia-reperfusion. $J$ Exp Med 2005; 201: 1135-1143.

27. Klebanoff SJ. Myeloperoxidase: friend and foe. J Leukoc Biol 2005; 77: 598-625, doi: 10.1189/jlb.1204697. 